\title{
RENOVAÇÃO DO QUALIS: EXEMPLO DA QUÍMICA
}

Qualis é uma lista de veículos utilizados para a divulgação da produção intelectual dos programas de pós-graduação stricto sensu (Mestrado e Doutorado). A Capes utiliza o Qualis para fundamentar o processo de avaliação do Sistema Nacional de Pós-Graduação.

O Conselho Técnico Científico (CTC) reiterou em 2005 o papel do Qualis para a fundamentação do processo de avaliação da pósgraduação nacional e destacou ser imprescindível, para o cumprimento das finalidades a que ele se propõe, a observância, por todas as áreas, do caráter classificatório do instrumento. "O Qualis de cada área deve expressar a classificação dos veículos utilizados nas categorias previstas, não devendo tal classificação se concentrar em uma ou duas categorias."

Entretanto, mesmo com estas orientações grande parte das áreas continha uma concentração excessiva, acima de $30 \%$ das publicações em, principalmente, veículos classificados como internacionais A (Tabela 1S, material suplementar). Nestes casos nitidamente não havia Qualis e a área estava simplesmente contando artigos (ou a área é uma exceção mundial e só publica nos melhores veículos). Esta concentração artificial da qualidade distorcia e não permitia a comparação de desempenho de Programas nem mesmo dentro de uma grande área. Estas distorções resultavam das diferentes interpretações que as áreas atribuíam aos critérios para as diferentes classificações (internacional, nacional e local) e até mesmo do valor relativo (para algumas áreas um artigo publicado em veículo nacional A valia mais que um publicado em internacional C). Havia extremos inaceitáveis como no caso de artigo publicado em veículo claramente de circulação nacional sem corpo de arbitragem que era classificado por uma área como local $\mathrm{C}$ e por outra como internacional A. Para algumas áreas o universo de veículos estava restrito àqueles onde a área publica e não aos existentes. Outras áreas empregavam a média ou mediana de algum indicador (como o FI, por exemplo) para delimitar os veículos internacionais A, resultando numa concentração artificial das publicações em um ou dois níveis. Algumas áreas nem mais empregavam os níveis nacional e local para classificar os periódicos. No triênio 2004-2006, 38\% das publicações por todas as áreas foram em periódicos Qualis internacional A (Tabela 1S). Em suma, o único ponto em comum a todas as áreas é que internacional A era sempre o mais elevado. Para algumas áreas, o segundo estrato mais elevado podia ser o internacional B ou o nacional A, enquanto outras igualavam nacional A e internacional A.

Isto criava uma dificuldade de compreensão na classificação de determinada área, por membros de outras áreas. O que se conclui é que a classificação vertical dos periódicos - praticada no triênio 20042006 - difere muito entre as áreas, tornando quase incompreensível, para uma área, o que a outra quer dizer quando localiza um periódico em determinado estrato.

Sendo assim, o CTC-CAPES constituiu uma comissão para discutir um balanço do Qualis de periódicos, apontando seus pontos positivos e sugerindo alterações. A comissão do Qualis foi presidida pelo Prof. Jairton Dupont (UFRGS), Coordenador da área de
Química. O CTC-CAPES deliberou, com o acordo das coordenações de áreas que nele não têm assento, para tornar o Qualis um instrumento mais robusto, transparente e que permita empregá-lo como um dos indicadores de comparação mais próxima do real desempenho em termos de produção científica qualificada entre as áreas, estabelecer uma única hierarquia vertical, que comporta A-1 como seu topo, seguido de A-2, B-1 até B-5 e finalmente $\mathrm{C}$, que é o estrato de valor zero. O objetivo é procurar uma linguagem comum, com novo Qualis que permita a qualquer pessoa de determinada área entender a avaliação de qualquer outra área. A classificação dos veículos se processa para qualquer área empregando os seguintes critérios básicos e comuns a todas áreas:

a) o(s) critério(s) de classificação dos veículos deve(m) levar em consideração todos os veículos disponíveis e não somente aqueles em que a área eventualmente publica;

b) veículos não indexados em alguma base de dados, sem corpo editorial e sem sistema de avaliação pelos pares devem ser classificados por todas as áreas como impróprio (C). Serão classificados de A (1,2) a B (1-5) somente os veículos indexados em alguma base de dados, com corpo editorial e com avaliação pelos pares (pareceristas ad hoc).

A área de Química classificou os periódicos de acordo (principalmente) com o fator de impacto (FI), com os respectivos pesos:

A1 - periódicos com FI igual ou superior a 4,0 (PESO 10);

A2 - FI inferior a 4,0 mas igual ou superior a 3,0 (PESO 5);

B1 - FI inferior a 3,0 mas igual ou superior a 2,0 (PESO 3);

B2 - FI inferior a 2,0 mas igual ou superior a 1,5 (PESO 2);

B3 - FI inferior a 1,5 mas igual ou superior a 1,0 (PESO 1);

B4 - FI inferior a 1,0 mas igual ou superior a 0,5 (PESO 0,5);

B5 - FI inferior a 0,5 mas igual ou superior a 0,1 (PESO 0,25).

Os periódicos Journal of the Brazilian Chemical Society, Química Nova e Anais da Academia Brasileira de Ciências serão classificados como A2, B1 e B1, respectivamente, independente de seus FI, devido à importância estratégica dos mesmos para a área de Química no país. Periódicos internacionais novos ou resultantes de fusão de dois ou mais títulos ou ainda de mudança de denominação, mas ainda não encontrados no JCR serão avaliados pela Comissão e receberão inicialmente a conceituação máxima de B1, de acordo com a origem inicial da revista com ajuda da Comunidade e dependendo das informações disponíveis sobre corpo editorial, editora e outros quesitos semelhantes. Devido às diferenças de FI entre os veículos das diferentes sub-áreas da Química e visando harmonizar o sistema, alguns periódicos independente de seus FIs serão classificados em estratos superiores (Tabela $2 \mathrm{~S}$ ).

O Qualis deve ter papel indutor, dizendo onde se deve publicar, não se limitando a avaliar onde se publicou. O padrão do novo Qualis deve ser padrão internacional de qualidade, contendo nos estratos superiores (A1 e A2) os periódicos reconhecidos pela comunidade internacional como os mais importantes e prestigiosos. 
O CTC recomenda o preenchimento de pelo menos cinco destes estratos, de modo que o Qualis satisfaça seu papel de poderoso sinalizador e indutor de quais são os periódicos mais importantes e quais os menos importantes para determinada área. Não há equivalência automática dos Qualis de uma área para outra e as áreas de produção mais consolidada não serão prejudicadas ao ocuparem, com seus periódicos de qualidade, também os estratos B. A simulação dos critérios da Química com os dados do triênio anterior (2004-2006) leva a uma distribuição equilibrada entre os sete estratos (Figura 1).

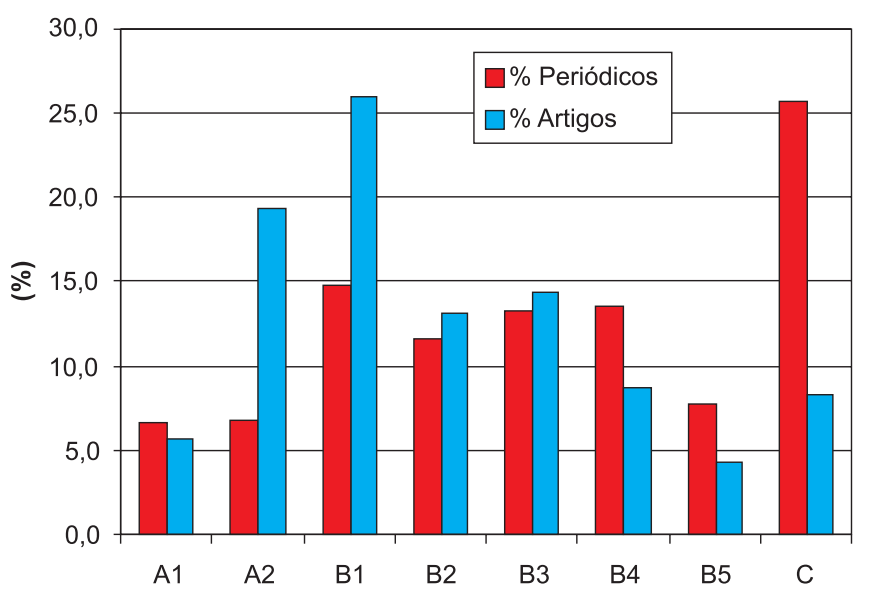

Figura 1. Distribuição dos periódicos e artigos da Área de Química publicados no triênio 2004-2006 em função do novo Qualis
Os periódicos onde a área mais publica cobrem quase todos os estratos (Tabela $3 \mathrm{~S}$ ).

Com estas definições poderemos melhor comparar as classificações dentre diferentes áreas e ter uma visão mais realista da inserção e impacto dos veículos em que estão sendo publicados os resultados de dissertações e por cada uma das áreas. Além disto, poderemos verificar como as áreas estão calibrando os indicadores que definem o Qualis. Felizmente, com os dados disponíveis e com os critérios empregados por cada uma das áreas poderemos entender e comparar como cada uma das áreas classifica os veículos em que os discentes/docentes estão publicando seus trabalhos, isto é, transformando definitivamente em científicos (passíveis de serem reproduzidos e reconhecidos pelos pares) os resultados de dissertações e teses. Não menos importante, o CTC voltará a dispor de um poderoso instrumento (indicador) de desempenho qualitativo e passível de ser empregado na comparação inter-áreas.

\section{MATERIAL SUPLEMENTAR}

As Tabelas 1S a 3S estão disponíveis em http://quimicanova.sbq. org.br, na forma de arquivo PDF, com acesso livre.

Jairton Dupont (IQ-UFRGS)

Coordenador da Área de Química

Luiz Carlos Dias (IQ-UNICAMP) Coordenador Adjunto da Área de Química 
Jairton Dupont (IQ-UFRGS)

Coordenador da Área de Química

Luiz Carlos Dias (IQ-UNICAMP)

Coordenador Adjunto da Área de Química

Tabela 1S. Distribuição porcentual dos artigos completos publicados em periódicos por estrato Qualis segundo as áreas de avaliação 2004-2006

\begin{tabular}{|c|c|c|c|c|c|c|c|c|c|}
\hline \multirow[t]{2}{*}{ Área de Avaliação } & \multicolumn{3}{|c|}{ Internacional } & \multicolumn{3}{|c|}{ Nacional } & \multicolumn{3}{|c|}{ Local } \\
\hline & IA & IB & $\mathrm{IC}$ & NA & $\mathrm{NB}$ & $\mathrm{NC}$ & LA & LB & $\mathrm{LC}$ \\
\hline Física & 62 & 18 & 20 & & & & & & \\
\hline Geociências & 31 & 17 & 2 & 30 & 12 & 8 & & & \\
\hline Mat/Estat. & 61 & 27 & 7 & 0 & 1 & 4 & & & \\
\hline Química & 41 & 35 & 15 & 2 & 2 & 2 & 2 & 0 & 1 \\
\hline Biol I & 57 & 11 & 5 & 10 & 8 & 7 & & & 2 \\
\hline Biol II & 81 & 15 & 4 & & & & & & \\
\hline Biol III & 53 & 20 & 16 & 1 & 0 & 9 & 0 & 0 & 1 \\
\hline Ecologia & 37 & 5 & 10 & 23 & 12 & 12 & 0 & 0 & 0 \\
\hline Computação & 60 & 24 & 7 & 0 & 9 & 0 & & & \\
\hline Engs I & 26 & 10 & 1 & 22 & 14 & 7 & 15 & 6 & 1 \\
\hline Engs II & 73 & 2 & 4 & 9 & 6 & 2 & 3 & 0 & 1 \\
\hline Engs III & 40 & 10 & 9 & 8 & 15 & 2 & 16 & 0 & \\
\hline Engs IV & 66 & 3 & 9 & 12 & 1 & 6 & 0 & 0 & 3 \\
\hline Educ. Física & 11 & 4 & 29 & 4 & 19 & 35 & ---- & ---- & --- \\
\hline Enfermagem & 5 & 29 & 43 & 1 & 11 & 11 & --- & ---- & --- \\
\hline Farmácia & 68 & 12 & 10 & 3 & 3 & 5 & & & \\
\hline Méd I & 42 & 13 & 23 & 6 & 13 & 4 & & & \\
\hline Méd II & 40 & 15 & 12 & 14 & 13 & 6 & 0 & & \\
\hline Méd III & 32 & 3 & 21 & 15 & 21 & 6 & 0 & 0 & 2 \\
\hline Odonto & 24 & 5 & 15 & 8 & 30 & 14 & 0 & 1 & 3 \\
\hline S Coletiva & 46 & 1 & 23 & 6 & 10 & 13 & 0 & 0 & 1 \\
\hline Agrar I & 28 & --- & 0 & 43 & 24 & 4 & --- & --- & --- \\
\hline C Alimentos & 32 & 8 & ---- & 38 & 11 & 10 & --- & --- & --- \\
\hline Méd Vet & 43 & --- & --- & 31 & 19 & 7 & --- & --- & --- \\
\hline Zoot\&RPesq. & 53 & --- & ---- & 29 & 15 & 3 & --- & --- & --- \\
\hline Adm\&Tur & 2 & 4 & 3 & 53 & 20 & 7 & 1 & 4 & 6 \\
\hline Arq\&Urb & 11 & 4 & 2 & 44 & 21 & 8 & 2 & 5 & 4 \\
\hline Direito & 6 & 8 & 3 & 16 & 12 & 7 & 48 & 0 & 0 \\
\hline Economia & 8 & 4 & 9 & 25 & 18 & 14 & 14 & 1 & 8 \\
\hline PlnjUrb\&Dem & 17 & 4 & --- & 28 & 20 & 1 & 5 & 12 & 13 \\
\hline Soc Aplic I & 0 & 5 & 3 & 42 & 18 & 16 & 7 & 4 & 5 \\
\hline SSoc\&EcoDom & 8 & 3 & 5 & 42 & 12 & 6 & 2 & 3 & 19 \\
\hline Antropologia & 27 & 3 & 1 & 21 & 13 & 8 & 19 & 4 & 4 \\
\hline C Política & 15 & 6 & 12 & 18 & 17 & 12 & 12 & 3 & 5 \\
\hline Educação & 13 & 3 & 5 & 15 & 17 & 24 & 8 & 7 & 9 \\
\hline Filosofia & 2 & 9 & 6 & 29 & 13 & 16 & 26 & --- & --- \\
\hline Geografia & 9 & 14 & 5 & 23 & 14 & 12 & 4 & 5 & 14 \\
\hline Historia & 20 & 5 & 9 & 20 & 11 & 11 & 7 & 7 & 9 \\
\hline Psicologia & 23 & 5 & 3 & 34 & 10 & 11 & 3 & 2 & 10 \\
\hline Sociologia & 9 & 6 & 3 & 15 & 15 & 21 & 11 & 8 & 11 \\
\hline Teologia & 1 & 13 & 7 & 12 & 27 & 17 & 22 & --- & --- \\
\hline Artes\&Musica & 4 & 3 & 1 & 38 & 36 & 3 & 7 & 6 & 2 \\
\hline Letras\&Ling. & 2 & 3 & 5 & 21 & 21 & 15 & 6 & 10 & 17 \\
\hline Ed. Ciên\&Mat. & 12 & 9 & 7 & 18 & 23 & 13 & 0 & 6 & 11 \\
\hline Multidisc. & 24 & 5 & 7 & 17 & 14 & 19 & 2 & 6 & 7 \\
\hline Soma & 38 & 10 & 10 & 16 & 12 & 8 & 3 & 1 & 2 \\
\hline
\end{tabular}

${ }^{\mathrm{a}}$ Fonte: CAPES 
Tabela 2S. Lista dos periódicos classificados no Qualis-Química independente do FI

\begin{tabular}{|c|c|c|c|}
\hline Entrada & Periódico & FI (2007) & Qualis $^{\mathrm{a}}$ \\
\hline 1 & Current Organic Chemistry & 3,961 & A1 \\
\hline 2 & Journal of Organic Chemistry & 3,959 & A1 \\
\hline 3 & Organometallics & 3,833 & A1 \\
\hline 4 & Bioelectrochemistry & 2,992 & $\mathrm{~A} 2$ \\
\hline 5 & Dental Materials & 2,99 & $\mathrm{~A} 2$ \\
\hline 6 & Neurochemistry International & 2,975 & $\mathrm{~A} 2$ \\
\hline 7 & Critical Reviews Analytical Chemistry & 2,973 & A2 \\
\hline 8 & Rapid Commun Mass Sp & 2,971 & $\mathrm{~A} 2$ \\
\hline 9 & Spectrochim Acta B & 2,957 & A2 \\
\hline 10 & Electroanal & 2,949 & $\mathrm{~A} 2$ \\
\hline 11 & J Chromatogr B & 2,935 & $\mathrm{~A} 2$ \\
\hline 12 & Sensor Actuat B-Chem & 2,934 & $\mathrm{~A} 2$ \\
\hline 13 & Free Radical Res & 2,925 & $\mathrm{~A} 2$ \\
\hline 14 & J Phys Chem A & 2,918 & A2 \\
\hline 15 & Eur J Org Chem & 2,914 & $\mathrm{~A} 2$ \\
\hline 16 & Tetrahedron & 2,869 & $\mathrm{~A} 2$ \\
\hline 17 & Electrochim Acta & 2,848 & $\mathrm{~A} 2$ \\
\hline 18 & Eur J Inorg Chem & 2,597 & $\mathrm{~A} 2$ \\
\hline 19 & Magn Reson Mater Phy & 1,494 & $\mathrm{~B} 2$ \\
\hline 20 & J Chem Educ & 0,56 & $\mathrm{~B} 2$ \\
\hline 21 & Quimica Nova na Escola & ----- & $\mathrm{B} 2$ \\
\hline 22 & Polímeros & ----- & $\mathrm{B} 2$ \\
\hline 22 & Acta Amazonica & ----- & $\mathrm{B} 2$ \\
\hline 22 & Revista Brasileira de Farmacognosia & ----- & $\mathrm{B} 2$ \\
\hline
\end{tabular}

a O próximo triênio a ser avaliado é 2007-2009 e a avaliação CAPES será em 2010. O fator de impacto a ser utilizado será o de 2008 pois o Qualis será definido em Maio de 2010. Entretanto, se a definição do Qualis for após o mês de Junho de 2009, empregaremos o Fator de Impacto de 2009. 
Tabela 3S. Lista dos 20 periódicos onde os PGs de Química mais publicaram em 2007a

\begin{tabular}{|c|c|c|c|c|}
\hline Clas. & Periódico & FI (2007) & Qualis $^{\mathrm{b}}$ & Artigos \\
\hline 1 & Journal of the Brazilian Chemical Society & 1,539 & $\mathrm{~A} 2$ & 474 \\
\hline 2 & Química Nova & 0,91 & B1 & 427 \\
\hline 3 & Journal of Thermal Analysis and Calorimetry & 1,483 & B3 & 150 \\
\hline 4 & Talanta & 3,374 & $\mathrm{~A} 2$ & 139 \\
\hline 5 & Acta Crystallographica. Section E & 0,508 & B4 & 105 \\
\hline 6 & Inorganica Chimica Acta & 1,713 & B2 & 103 \\
\hline 7 & Tetrahedron Letters & 2,615 & B1 & 99 \\
\hline 8 & Journal of Colloid and Interface Science & 2,309 & B1 & 89 \\
\hline 9 & Electrochimica Acta & 2,848 & $\mathrm{~A} 2$ & 88 \\
\hline 10 & Journal of Molecular Structure & 1,486 & B2 & 88 \\
\hline 11 & $\begin{array}{l}\text { Acta Crystallographica. Section C, Crystal Structure } \\
\text { Communications }\end{array}$ & 0,719 & B4 & 81 \\
\hline 12 & Chemical Physics Letters & 2,207 & B1 & 77 \\
\hline 13 & Analytica Chimica Acta & 3,186 & $\mathrm{~A} 2$ & 76 \\
\hline 14 & Journal of Molecular Catalysis. A, Chemical & 2,707 & B1 & 75 \\
\hline 15 & Journal of Physical Chemistry. B & 4,086 & A1 & 75 \\
\hline 16 & Eclética Química (Araraquara) & 0,000 & B5 & 68 \\
\hline 17 & International Journal of Quantum Chemistry & 1,368 & B3 & 67 \\
\hline 18 & $\begin{array}{l}\text { Spectrochimica Acta. Part A, Molecular and Biomolecular } \\
\text { Spectroscopy }\end{array}$ & 1,511 & B2 & 66 \\
\hline 19 & Journal of Chromatography & 3,641 & $\mathrm{~A} 2$ & 65 \\
\hline 20 & Microchemical Journal & 1,8 & B2 & 65 \\
\hline
\end{tabular}

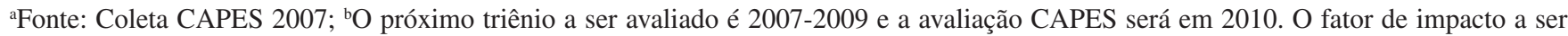
utilizado será o de 2008 pois o Qualis será definido em Maio de 2010. Entretanto, se a definição do Qualis for após o mês de Junho de 2009, empregaremos o Fator de Impacto de 2009. 